\title{
Dinâmica de populações aplicada ao cultivo da carpa comum - Capacidade de suporte
}

\author{
Anastácio Castelo Matos ${ }^{1}$ e Ângelo Paggi Matos²
}

\begin{abstract}
Resumo - Este trabalho tem por objetivo fornecer informações técnicas sobre a dinâmica de população aplicada ao policultivo de carpas para maximização da produção de biomassa. Nesta nota técnica, apresentamos a capacidade de suporte aplicada no cultivo da carpa comum (Cyprinus carpio) cultivada em sistema de policultivo em viveiros escavados (área de $250 \mathrm{~m}^{2}$ ) com e sem a presença de aeração. Para tanto, é necessário o conhecimento prévio de algumas variáveis, tais como: viveiro-área, espécie cultivada, densidade populacional, população inicial, data de peixamento, data de despesca, produção total do viveiro, taxa de sobrevivência, conversão alimentar, percentagem de indivíduos na produção e duração do cultivo. A espécie de carpa comum quando cultivada no sistema com aeração, com densidade de 1 peixe $2 \mathrm{~m}^{2}$, apresenta no pico de 27,8 meses uma biomassa máxima de 183.772 gramas $(183,77 \mathrm{Kg})$, sendo que é neste período que deve ser efetuada a despesca total, com tendência de lucro máximo.
\end{abstract}

Termos para indexação: Piscicultura; Policultivo de peixes; Estimativa de biomassa.

\section{Population dynamics applied to common carp farming - Carrying capacity}

\begin{abstract}
The aim of this study is to provide useful information about the population dynamics applied to carp cultivation for maximum biomass production. In this report, the carrying capacity of common carp (Cyprinus carpio) grown on polyculture system in earthen ponds $\left(250 \mathrm{~m}^{2}\right.$ pond-area) with aeration and without aeration was investigated. Prior knowledge of pond surface area, fish species cultivated, initial and density population, survival rate, feed conversion, cultivation time, and total fish production are some key variables required to estimate fish farming carrying capacity. Common carp when grown on aerated system, with density population of 1 fish $2 \mathrm{~m}^{2}$, has produced a maximum biomass yield of $183,772 \mathrm{~g}(183.77 \mathrm{Kg})$ achieved at the peak of 27.8 months, suggesting that total fish harvesting in this period of time is highly recommended due to its maximum profitability.
\end{abstract}

Index terms: Fish farming; Polyculture of fish; Biomass estimation.

\section{Introdução}

A análise da dinâmica de populações é um estudo básico em vários campos das ciências biológicas, como em ecologia, genética de populações, parasitologia, epidemiologia, etc., e em projetos de erradicação, preservação e exploração de populações naturais.

Para um estudo de populações, alguns conceitos são importantes, sendo que a população pode ser dividida em:

- População biológica: É o conjunto de indivíduos da mesma espécie que vivem na mesma área. É a população que é estudada nas suas características e fenômenos que ali ocorrem, por amostragem, para podermos estender a população de um modo racional.

- População estatística: É o conjunto de eventos expressados por valores numéricos. É uma população estimada por meio de modelos matemáticos.

\section{Aquicultura quantitativa}

Em uma piscicultura intensiva, quando iniciada na fase não larval, as análises de gráficos por meio de modelos matemáticos são de suma importância para avaliar o rendimento da produção de peixes por área alagada, taxas de crescimento, curvas de biomassa, otimi- zação dos cultivos e fatores econômicos (MATOS, 1977). Para tanto, é indispensável o conhecimento dos seguintes parâmetros: viveiro-área $\left(\mathrm{m}^{2}\right)$, espécie (s) cultivada, densidade populacional, população inicial, data de peixamento, data de despesca, produção total do viveiro, taxa de sobrevivência, conversão alimentar, percentagem de indivíduos na produção e duração do cultivo (MATOS, 1996).

Com os dados das amostragens feitas mensalmente, podemos determinar a curva de biomassa de um dado viveiro em função do tempo. A curva da biomassa depende diretamente dos seguintes fatores: curva de crescimento 
em comprimento, relação peso/comprimento, curva de crescimento em peso e taxa de sobrevivência em um determinado tempo de cultivo. Os modelos matemáticos e as equações aplicadas à dinâmica de populações na piscicultura podem ser consultados na literatura publicada em Santos (1978). Gunkel et al. (2015), por sua vez, determinaram a capacidade de suporte do cultivo de tilápias (Oreochromis niloticus) em tanques-rede instalados em reservatórios na bacia do rio São Francisco, Brasil.

\section{Capacidade de suporte no cultivo da carpa comum (Cyprinus carpio)}

\section{- Metodologia}

Em um policultivo de peixes, sendo a carpa comum povoada na densidade de 1 peixe para cada $2 m^{2}$, ou seja, 5 mil peixes por hectare (ha), alimentação com ração convencional e duração do cultivo de 1 ano, é possível determinar a curva de biomassa para estimar a capacidade de suporte do viveiro. No presente estu- do de caso foram testados seis viveiros escavados com $250 \mathrm{~m}^{2}$ de área alagada e $1,5 \mathrm{~m}$ em média de profundidade.

Mensalmente foram amostrados $20 \%$ (25 peixes) em cada unidade experimental para obtenção dos valores de peso (g) e comprimento (cm) total individual, assim como estimar a biomassa total, conversão alimentar e ajuste de ração. Para tanto, utilizou-se uma rede de arrasto multifilamento para captura dos peixes, malha de $8 \mathrm{~mm}$ entre nós, medindo $25 \mathrm{~m}$ de comprimento e $3 \mathrm{~m}$ de altura. O comprimento dos peixes foi medido com um ictiômetro, precisão de $0,1 \mathrm{~cm}$, e o peso medido com uma balança digital da marca Marte, modelo AS 5000 com precisão de 0,1 grama.

O modelo de cultivo sem aeração apresentou uma variação de $\mathrm{O}_{2}$ entre 6,3 e $10,2 \mathrm{mg} \mathrm{L}^{-1}$, enquanto no modelo de cultivo com aeração os valores variaram de 7,5 a $11,6 \mathrm{mg} \mathrm{L}^{-1}$. Importante enfatizar que os valores de $\mathrm{O}_{2}$ para os dois modelos estudados foram máximos entre $14 \mathrm{~h} 00$ e $17 \mathrm{~h} 00$ da tarde, período em que ocorre maior processo fotossintético das algas.

Na Figura $1 \mathrm{~A}$ é possível visualizar a curva de crescimento em peso da carpa comum, sendo a Figura $1 \mathrm{~A}$ a curva de crescimento em peso para o modelo de cultivo sem aeração e Figura 1D para o modelo de cultivo com aeração.

Denominamos curva de biomassa a variação da biomassa de um viveiro em função do tempo. Para calcular a curva de biomassa é necessário determinar a taxa média mensal de sobrevivência. Conhecidos o número inicial $\mathrm{R}$ de indivíduos ( $R=125$ peixes) em uma área de $250 \mathrm{~m}^{2}$ e a taxa média mensal de sobrevivência (95\%), é possível estimar a curva de biomassa total, que está apresentada nas Figuras 1B (sem aeração) e $1 \mathrm{E}$ (com aeração).

\section{- Modelo de cultivo sem aeração}

Analisando a Figura 1B na curva de biomassa, no modelo de cultivo sem aeração, conclui-se que na idade (T) de 22,7 meses, o instante de biomassa máxima é de 104.024,2 gramas (104,02Kg), o que caracteriza a capacidade de suporte do sistema, decaindo posteriormente após este período. Contudo, o
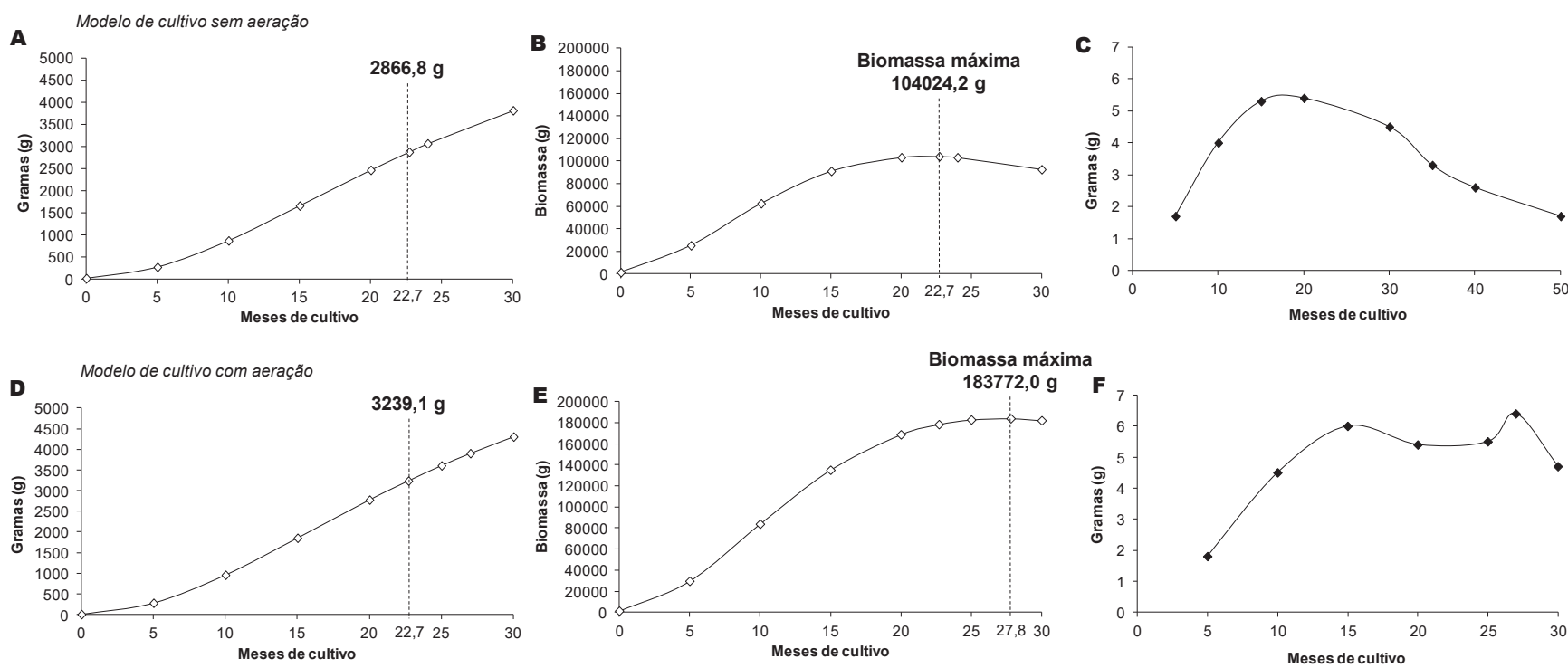

Figura 1. Dinâmica de população da carpa comum (Cyprinus carpio) cultivadas em viveiros escavados. Modelo de cultivo sem aeração (A-C) e modelo de cultivo com aeração (D-F); $A$ e D - comparação da curva de crescimento em peso (g) em função dos meses de cultivo sem e com aeração; B e E - comparação curva de biomassa total definindo a capacidade de suporte do sistema; C e F - comparação do ganho de peso (g/mês) ao longo do cultivo

Figure 1. Population dynamics of common carp (Cyprinus carpio) cultivated in earthen ponds. Cultivation mode without aeration (A-C) and aerated (D-F); $A$ and $D$ - comparison of growth curve in terms of weight ( $g$ ) as a function of cultivation months; $B$ and $E$ - comparison of total biomass that define the maximum carrying capacity of the system; C and F-comparison of weight gain ( $\mathrm{g} / \mathrm{month}$ ) during cultivation 
cultivo foi conduzido por somente 12 meses, e nesta data (12 meses), a biomassa obtida (despesca) foi de $80.263,7$ gramas $(80,26 \mathrm{Kg})$ e, portanto, bem inferior à biomassa máxima estimada, que é $104.024,2$ gramas $(104,02 \mathrm{Kg})$ no tempo de 22,7 meses. Assim, fica evidente que o cultivo deveria ter sido conduzido por mais 10,7 meses, para então se obter a máxima produtividade em biomassa no viveiro.

O gráfico da Figura $1 \mathrm{C}$ ilustra o ganho de peso, grama/mês, ao longo do cultivo sem aeração. Observa-se que o maior ganho de peso ocorre justamente de 20 a 22 meses de cultivo, com o máximo de $5,4 \mathrm{~g} \mathrm{dia}^{-1}$, e os menores são no início do cultivo com $1,7 \mathrm{~g} \mathrm{dia-1}$. Portanto, se projetarmos para 50 meses, há um ganho também de $1,7 \mathrm{~g} \mathrm{dia}{ }^{-1}$.

\section{- Modelo de cultivo com aera-} ção

Seguindo o mesmo modelo anterior, e com a mesma densidade de povoamento, 125 peixes $/ 250 \mathrm{~m}^{2}$ (5000 ha-1); sobrevivência de 96\%, assim como todos os outros parâmetros calculados, observa-se que no mesmo período de 22,7 meses houve um acréscimo de biomassa de $372,36 \mathrm{~g}$ mês $^{-1}$ ou $12,99 \%$ a mais do viveiro aerado em relação ao não aerado (Figura 1D).

Assim, a capacidade de suporte em biomassa do viveiro aerado foi de 183.772 gramas $(183,77 \mathrm{Kg})$ no período de 27,8 meses, sendo que a melhor época para a realização total da despesca dos peixes é neste período mensal, com tendência de lucro máximo. De forma comparativa, pode-se afirmar que o estudo com 12 meses de duração mostrou um ganho de biomassa somente de $100.489,7$ gramas $(100,48 \mathrm{Kg})$, ou seja, $82,9 \%$ a menos em relação à biomassa total final. Ademais, o crescimento em biomassa calculada do viveiro aerado foi $76,7 \%$ superior ao não aerado, ilustrando o ganho de biomassa significativo quando se utiliza nos viveiros de peixes um sistema de aeração.

Por fim, é possível visualizar na Fig- ura $1 \mathrm{~F}$ que o ganho de peso/mês do viveiro aerado foi bastante alterado, com picos em 15 meses com 6,0g dia ${ }^{-1}$, e tendo o máximo justamente no 27 mês com 6,4g dia-1, decrescendo posteriormente nos meses seguintes.

\section{Otimização do rendimento em biomassa do cultivo da}

\section{carpa comum em sistema aerado e sem aeração}

Lançando-se $\mathrm{I}_{\mathrm{B}}=$ índice de rendimento de biomassa contra o número de indivíduos (R), em viveiros idênticos, obtém-se o valor ótimo (Otimização = $\mathrm{R} \times \mathrm{I}_{\mathrm{B}}$ ) de produção máxima sustentável (Figura 2A e B).

Na Figura $2 A$ é possível verificar que o índice de biomassa do viveiro não ae- rado é de 4.600 gramas em comparação ao sistema aerado que foi de 6.600 gramas. Além disso, a produção máxima sustentável da carpa comum em viveiros sem aeração foi de $575.000 \mathrm{~g}$, valor este menor do que o viveiro com aeração (825.000g) (Figura 2B), ilustrando que o efeito da aeração tem influência direta no crescimento e na produção de peixes.

É importante considerar que nestes dois modelos de cultivos, ou seja, aerado e não aerado, em um sistema de policultivo de peixes, sendo a carpa comum a espécie principal e alimentadas com rações balanceadas, os crescimentos em comprimento e peso foram significativos. Lembrando que a densidade de povoamento da carpa comum deve ser de 1 peixe $2 \mathrm{~m}^{2}$ de área alagada, mas
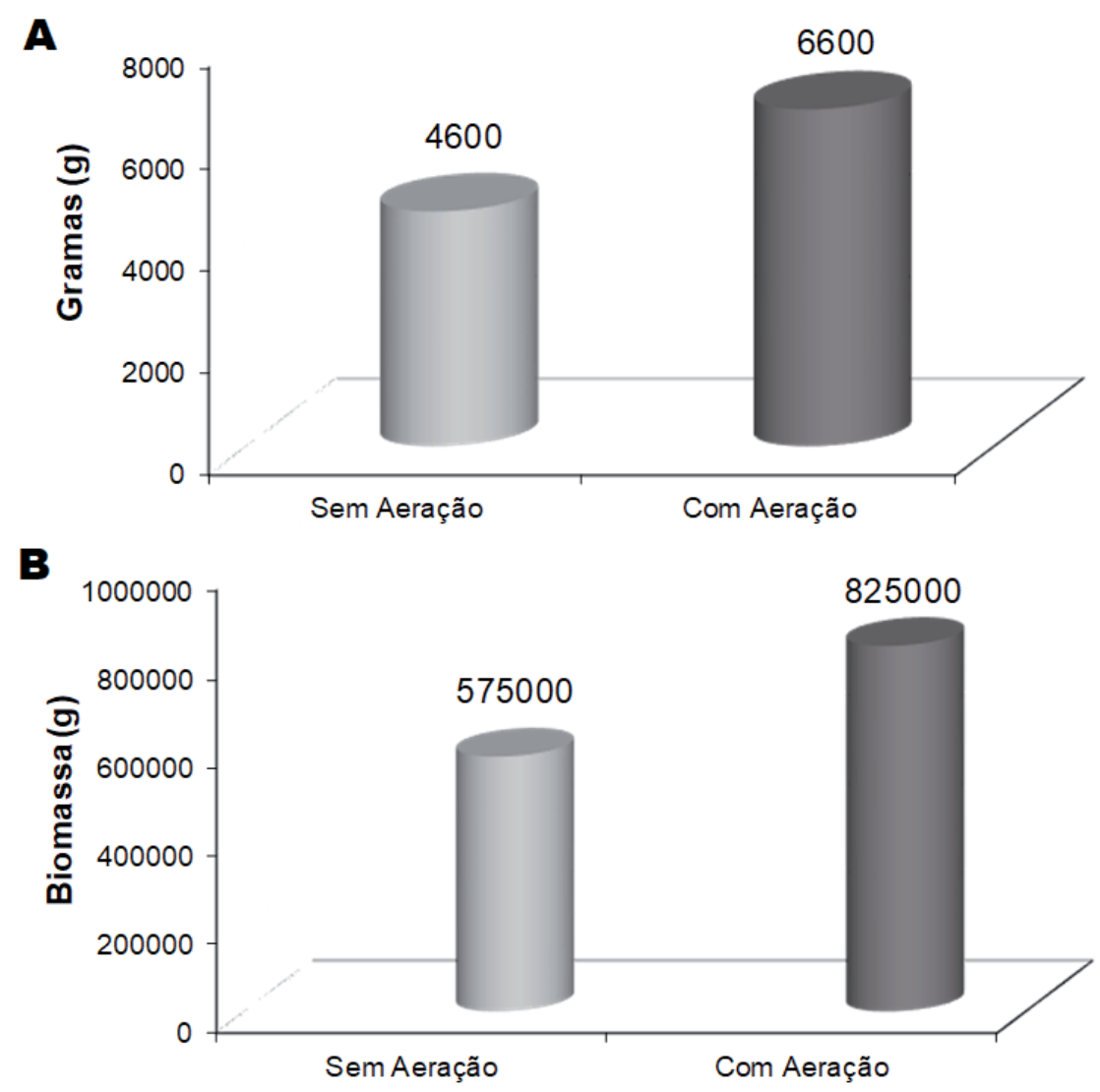

\section{Viveiros}

Figura 2. Rendimento em gramas da carpa comum cultivada em viveiro escavado sem e com aeração. A - Índice de rendimento em biomassa; B - Otimização da produção de biomassa.

Figure 2. Yield of common carp cultivated in earthen pond with and without aeration. AIndex of biomass yield; $B$ - Optimization of biomass production. 
Tabela 1. Parâmetros que devem ser avaliados para predizer a capacidade de suporte do cultivo da carpa comum em viveiros escavados

Table 1. Parameters to be considered in order to estimate the carrying capacity of common carp cultivated in earthen ponds

Variável

Área do viveiro
Unidade

$\mathrm{m}^{2}$

$\mathrm{cm}$

gramas

$5.819,4$

22,7

meses

gramas

103.900

183.200
Viveiro não

Viveiro

aerado
Instante de biomassa máxima

Biomassa máxima

gramas

4.600

6.600

Índice de rendimento em biomassa

quilogramas

4.156

7.328

Biomassa máxima para 1 hectare (extrapolação)

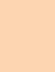

Observação: 1 peixe para cada 2 m2 (5.000 ha-1)

o peixamento total em um sistema de policultivo pode variar de 1 peixe para cada 1 metro quadrado $\left(1 \mathrm{~m}^{2}\right)$ até 1 peixe para cada $5 \mathrm{~m}^{2}$ de área alagada, dependendo das espécies de peixes a serem cultivadas.

\section{Considerações adicionais} sobre o cultivo da carpa comum

Alguns dados numéricos importantes sobre estes dois modelos de cultivo, não aerado e com aeração no desenvolvimento da carpa comum estão exemplificados na Tabela 1.

\section{Considerações finais}

A aplicação da dinâmica de populações na piscicultura possibilita que se avaliem o ótimo da despesca máxima em relação ao tempo de cultivo e o lucro máximo de quaisquer espécies em cultivo. Para tanto, basta gerenciamento no empreendimento.

\section{Referências}

GUNKEL, G.; MATTA, E.; SELGE, F.; Da SILVA, G.M.N.; SOBRAL, M.C. Carrying capacity limits of net cage aquaculture in Brazilian reservoirs. Brazilian Journal of Enviromental Sciences, v.36, p.128-144,
2015. DOI: https://doi.org/10.5327/ Z2176-947820151008.

MATOS, A.C. Dinâmica de Populações Aplicada à Aqüicultura. Monografia como parte das exigências para a obtenção do título de Engenheiro de Pesca. Universidade de São Carlos (UFSCar), São Carlos, SP, 1977.

MATOS, A.C. Efeito da Aeração e da Taxa de Renovação de Água em Policultivo de Peixes. Mestrado em Aquicultura, Universidade Federal de Santa Catarina (UFSC), 1996. 129p. Dissertação (Mestrado em aquicultura).

SANTOS, E.P. Dinâmica de populações aplicada à pesca e piscicultura. São Paulo, HUCITEC. Ed. Universidade de São Paulo (USP), 1978. 